\title{
Research on the Rapid Growth of the Chamber Effect on Social Media
}

\author{
ZiPeng Chen ${ }^{1, *}$ \\ ${ }^{1}$ vankeMeisha Academia, Shenzhen, GuangDong Province, 518000, China \\ *Corresponding author. Email: czp0312@qq.com
}

\begin{abstract}
The research is based on the phenomenon of constantly emerging echo chambers on social media that have created significant and huge damage to online communities. The formation of echo chambers seems to be unavoidable. The purpose of this paper is to discuss the development of modern echo chambers, especially on social media, and the existence of intentionally manipulated echo chambers used to gain profit or political power, such as "fake news". The research object is social media echo chambers, and it attempts to approach them and the social media users who are in echo chambers using psychology. The paper is simply based on research on the Internet with a possible experiment (a hypothesis) that could be carried out to discover if there is a necessary correlation between the attitude inside of an echo chamber effect and the growth of it. It is unclear for now whether the echo chamber's growth is relevant to attitudes, but it is certain that the echo chamber is gradually distorting views of users through group polarization and group think.
\end{abstract}

\section{Keywords: Echo Chamber Effect, Group Polarization, Social Media, Framing}

\section{INTRODUCTION}

The echo chamber effect, described as "an environment where a person only encounters information or opinions that reflect and reinforce their own. Echo chambers can create misinformation and distort a person's perspective so they have difficulty considering opposing viewpoints and discussing complicated topics. They're fueled in part by confirmation bias, which is the tendency to favor info that reinforces existing beliefs." [1]. The main components are limited information or misinformation, distorted perspective and tendency to favor. Under an environment with information only from one perspective could easily lead somebody onto the same path. When one person makes a point, the others are going to be influenced by him just like how the theory of "framing" is. As described "when our decisions are influenced by the way information is presented"[2]. When others receive the opinion and made one that's aiming for the same direction, more would be influenced. The echo chamber later is formed and the same voice is spread and bounced back to the one making the voice again and again while the whole emotion is taken to another level, eventually become intense and distorted.

\section{BACKGROUND}

Before the actual term of Echo chamber, which specifically describes how ideas get extreme within a group on social media, the phenomenon is known as "group polarization"[6]. Echo chamber has been around for a long time, as a normal thing when information transferring was still difficult, as political tools used by the controllers to have the citizens' views under control like what used to happen in the Qing Dynasty and the Soviet Union. Messages were difficult to be delivered even just from one city to another, otherwise, the story of Marathon would not have existed. Messages are limited either due to technical, transportation issues or the local governments are intentionally controlling a specific proportion of the information from their people, forming echo chambers. If it's used successfully, then hopefully patriotism would be developed among the citizens. When it is distorted, blind worship and confidence flourish, pushing people to the extreme.

This could now be seen everywhere on social media. Facebook, Twitter, Instagram, Weibo and Tik-tok, are all the hotbeds of echo chambers and also the hotbeds of the chaotic, toxic environment on the World Wide Web. We can say that the invention of social media has taken 
the echo chamber effect to its peak, and unfortunately, it is unintentional, meaning that it could not be stopped with the development of the information age.

Countless echo chambers are formed while people are holding different views on countless topics. Political preferences, human rights issues, celebrity preferences, subcultures, racism, and even trivialities like gaming console preferences are all new emerging echo chambers on social media. Whenever a political idea is expressed, a human rights issue is brought to the stage, a new subculture has been developed, another echo chamber jumps into the vision of the society. There would be positive effects, but everything goes bad whenever it goes too far. When central ideas are distorted, the true face of the echo chamber effect emerges, bringing the online society down a notch.

\section{ANALYSIS OF THE ECHO CHAMBER EFFECT}

The true essence is, echo chambers on social media are nothing like those that existed before. It is still echo chamber, but is now reborn as a byproduct of social media which has been having effects on people's views on numerous issues and the actual impact could not be measured. What used to happen was caused by limited information and resources yet what is happening now on the World Wide Web is more of a combination of both limited information and the fact that people are no longer accepting views from the other side. People are not "mentally ready" or simply can't stand others who are having opposite views when them.

Echo chambers have their good sides, it assembles those who have the same ideas together to spread their opinions and love towards something. However, these echo chambers are always double-edged blades. As described before, while the voice is bouncing back and forth inside the echo chamber, opinions made by one person come back to one from the others. These central ideas of opinions are all modeled the same though they could have appeared different from the outside. More people getting into the echo chamber, more voices with the same perspective gather together, forming a bigger voice, enhancing the idea, gradually distorting it to a moment that the echo chamber can no longer hold. The ideas were repeated over and over again.

Not surprisingly, the different ways of the perspective are carried out gradually change the direction of the voice. The voice is now distorted, might look nothing like what it used to be. This is quite common when it comes to political views and chasing celebrities. Eventually, the echo chamber would start to influence the outside world, echo chambers encounter with each other, collapsing, ideas exchanging, each idea accepted or not, there is always going to be some negative effect.
It is common to see people online fight over these topics, this occurs every second on the social media, whenever a point is made there's going to be someone from another echo chamber that has a different view of who's going to spread the value and perspective this person holds and eventually become conflicts of two echo chambers. This is the result of echo chamber rooms' growth, when those voices get out of the chamber and run into others. When the social media is not big enough for echo chambers, they are eventually going to collide and conflicts emerge simultaneously.

\section{SOCIAL MEDIA ALGORITHM- THE FILTER BUBBLE}

Another concept of an echo chamber is known as a filter bubble. Just like its name, a filter bubble is known as filtering opposing ideas. "...it becomes difficult to have a meaningful discussion about the facts. This is how filter bubbles contribute to a lack of understanding and an unwillingness to consider opposing viewpoints and unfavorable information."[1][2]. As the concept's creator Eli Pariser claimed, being in a filter bubble means that the algorithms on the social media are likely to block a user from ideas that haven't expressed any interest by the user or simply just the opposite of what the user usually looks at. The user is isolated by the algorithms and are less likely to get in contact with information on the other side of the lawn. The lack of information and the misunderstanding later developed strengthens the echo chamber, which is also why it is effectively influencing every single user possible.

\section{INTENTIONALLY BUILT ECHO CHAMBERS AND "FAKE NEWS"}

Echo chambers are formed either unintentionally or intentionally. Commonly, unintentional echo chambers, like sub-Reddits, Youtube comment sections and Instagram of a celebrity are naturally developed due to the same opinion or same love towards something just like described, even though these might also have negative effects and later become chaotic, they are probably not as terrible as some of the intentionally built echo chambers.

Echo chambers with negative attitudes or direction towards a topic are the most common type of echo chambers that are intentionally made by people. Fake news, or we could call them, is a huge echo chamber that has been around on social media to control the thoughts of its users. "The Internet is an incredible resource for news and information, but unfortunately not everything online is trustworthy. Fake news is an article or video containing untrue information disguised as a credible news source." [1][2]. Echo chambers, as described, could be manipulated by some as financial or political tools to gain advantages from users with 
limited information.Fake news and some of the bot spammers we see under comment sections of a product or a news page, are made by those who are trying to artificially building up an echo chamber.

The emergence of fake news is getting more and more frequently and intensely throughout the internet. Again, the purpose of fake news is to use the echo chamber effect to manipulate the thoughts of the users, meaning that even though the messages given might seem to be quite ridiculous for us, the consumers of these fake news might have no idea of the accuracy of these reports.

"A fake news article is designed to outrage and shock, causing some readers to share it on Facebook, Twitter, or another type of social media platform without questioning it.”[1]. Negative emotions are the biggest weapons of these fake news. As to the theory of positive psychology, negative emotions are way stronger than positive ones. One is probably going to feel stronger emotions looking at some made up irritating news than something like the National Geographic Magazine. "In fact, people ruminate about events that induce strong negative emotions five times as long as they do about events that induce strong positive ones." [4]. The fake news intentionally build up echo chambers with more negative attitudes (relatively, as all Echo Chambers are negative) towards what are out of the echo chamber and may also directly point at a target that the echo chamber is planning to criticize.

People are less likely to question news that they have little knowledge about, like news about foreign countries or new technologies and what's inside of a vaccine. As the flood of negative information has flooded the users of these fake news, the users would end up being "brain-washed"by the echo chamber. They would become part of it, helping the fake news to take to flood to their family and friends who are also limited with information.

\section{HOW TO AVOID ECHO CHAMBERS}

Even though echo chambers are everywhere through out the internet, it does not necessarily means that they could not be avoided. In the end, it is still a lack of information that leads to such a situation. Forbes provided five strategies to avoid echo chamebrs on social meida. According to Forbes, initially, awareness is needed for an individual to stay away from this type of brain-wash. Users need to be aware of confirmation bias that could be included in their information resources and should be looking for opinions that are relatively more objective, more neutral ideas just like the theory of framing described[5].

Different perspectives, ideas from diverse groups of people can also successfully help the users to keep themselves sober while looking at an issue. There might still be echo chambers, but it would be easier to discover an objective view when both sides of the issue is revealed. Eventually, there should be pre- and postanalysis of an issue. Echo chambers are the most effective to those who are easily led onto the path the echo chambers have platformed, meaning that analysis is essential for one to stay sober from the flawed opinions of these echo chambers.

\section{CONCLUSION}

Still, it is not clear that whether there is a cause and effect between the attitude of an echo chamber and the growth rate of an echo chamber. There is, however, a quite obvious correlation between negativity and the echo chamber effect just like how the "fake news" as described are, but there is not enough evidence nor any experimental designed research conducted to show there is necessarily a causal relationship between attitudes of an echo chamber and the growth of an echo chamber.

What could be done, is a quasi-experiment with a bot or a written program designed that is able to identify the attitudes towards a topic by identifying the words with attitudes -for example, a tweet including more negative words like "unpleasing" than positive terms "fabulous" can be categorized as a negative one- on social media. After numerous posts are categorized into negative and positive ones, the numbers of these posts on social medias would be counted each week within a month and the growth rate would be measured (population1-population0/population $0 * 100 \%$ ). Вy comparing the growth rate of the two categories, it could be concluded that whether attitudes towards a topic would have a causal relationship with the growth rate of an echo chamber.

Unavoidably, echo chambers are going to emerge and expand on the social medias and influence the views of millions. Unfortunately, the development of echo chambers could not be stopped and it is impossible to stay unaffected. It is important for Individuals to develop awareness towards echo chambers and to keep themselves informed through different resources. Ration, intelligence, knowledge, only these are able to stay sober under such mind traps.

\section{ACKNOWLEDGMENT}

Thanks to Professor Schiappa from MIT, providing me with extra information about the association between the Framing Effect and the Echo chamber effect. Also I have to express my thanks to my school AP Psychology teacher Ms.Maffei, informing about group polarization.

\section{REFERENCES}

[1] Dital Media Literac. What is an Echo Chamber? https://edu.gcfglobal.org/en/digital-media-literacy/ 
what-is-an-echo-chamber/1/Accessed

1 st ,2021.

[2] The Decision Hub(2021) What is the Framing Effect?

https://thedecisionlab.com/biases/framing-effect/Ac cessed Sep 1st ,2021.

[3] Matteo Cinell,Gianmacro De Francisci Morales, Alessandro galeazzi, Walter,Quattrociocchi, Michele Starnin(2021) The echo Chamber Effect on Social Media. https://www.pnas.org/content/118/9/e2023301118A ccessed Sep 13th,2021.

[4] Psycholgoy Today.(2010) Are Negative Emotions More Important than Positive Emotions? https://www.psychologytoday.com/intl/blog/in-thename-love/201007/are-negative-emotions-more-im portant-positive-emotions. Accessed Sep 15th, 2021.

[5] Forbes.(2021) Avoiding Echo Chamber: 5 Strategies To Beat Confirmation Bias. https:/www.forbes.com/sites/iese/2021/06/16/avoi ding-echo-chambers-5-strategies-to-beat-confirmati on-bias/?sh=cb19a0e1267d. Accessed Sep 16 th ,2021.

[6] Alvernia University. (2018), Group Polarization in Social Psychology https://online.alvernia.edu/articles/group-polarizati on-social-psychology/. Accessed Sep 16th ,2021. 